\title{
Detection of Late Gadolinium Enhancement on Cardiovascular Magnetic Resonance by Global Longitudinal Strain Using Speckle-Tracking Echocardiography in Patients with Nonischemic Cardiomyopathy
}

Shingo Ota

Wakayama Medical University

Takeshi Hozumi ( $\nabla$ takeshi.hozumi@gmail.com )

Wakayama Medical University https://orcid.org/0000-0002-6027-9053

Takashi Tanimoto

Wakayama Kenritsu Ika Daigaku

Kazushi Takemoto

Wakayama Kenritsu Ika Daigaku

Teruaki Wada

Wakayama Kenritsu Ika Daigaku

Manabu Kashiwagi

Wakayama Kenritsu Ika Daigaku

Kunihiro Shimamura

Wakayama Kenritsu Ika Daigaku

Yasutsugu Shiono

Wakayama Kenritsu Ika Daigaku

Akio Kuroi

Wakayama Kenritsu Ika Daigaku

Yoshiki Matsuo

Wakayama Kenritsu Ika Daigaku

Hironori Kitabata

Wakayama Kenritsu Ika Daigaku

Yasushi Ino

Wakayama Kenritsu Ika Daigaku

Takashi Kubo

Wakayama Kenritsu Ika Daigaku

Atsushi Tanaka

Wakayama Kenritsu Ika Daigaku 
Takashi Akasaka

Wakayama Kenritsu Ika Daigaku

\section{Research}

Keywords: global longitudinal strain, speckle-tracking echocardiography, late gadolinium enhancement, cardiovascular magnetic resonance, nonischemic cardiomyopathy

Posted Date: May 28th, 2020

DOI: https://doi.org/10.21203/rs.3.rs-31077/v1

License: (c) (i) This work is licensed under a Creative Commons Attribution 4.0 International License. Read Full License 


\section{Abstract \\ Background}

The presence of late gadolinium enhancement (LGE) using cardiovascular magnetic resonance (CMR), suggesting myocardial fibrosis is useful as a prognostic index in patients with nonischemic cardiomyopathy (NICM). The present study aimed to investigate whether left ventricular (LV) global longitudinal strain (GLS) using speckle-tracking echocardiography (STE) can be used as a surrogate marker for the detection of CMR-LGE in patients with NICM.

\section{Methods}

The study included 50 patients with NICM who underwent both STE and CMR. The presence, or absence of CMR-LGE was assessed in all patients, whereas STE-GLS was successfully analyzed in $41 / 50(82 \%)$ patients. Therefore, the final study population comprised these 41 patients. Patients were divided into those with CMR-LGE (Group A; $n=18$ ) and those without CMR-LGE (Group B; $n=23$ ). Echocardiographic indexes including GLS was compared between the two groups.

\section{Results}

No significant differences were observed in LV end-diastolic and end-systolic volume indexes, LV ejection fraction, mitral $\mathrm{E} / \mathrm{A}$, deceleration time, $\mathrm{E} / \mathrm{e}^{\prime}$, left atrial volume index, and the systolic transtricuspid pressure gradient between the Groups- $A$ and $B\left(113 \pm 30\right.$ vs. $109 \pm 24 \mathrm{~mL} / \mathrm{m}^{2}, 82 \pm 27 \mathrm{vs} .78 \pm 22 \mathrm{~mL} / \mathrm{m}^{2}$, $28 \pm 6$ vs. $29 \pm 7 \%, 1.3 \pm 1.1$ vs. $1.2 \pm 0.8,201 \pm 82$ vs. $214 \pm 69 \mathrm{~ms}, 11.3 \pm 3.7$ vs. $11.7 \pm 5.9,49 \pm 24$ vs. 49 $\pm 13 \mathrm{~mL} / \mathrm{m}^{2}$, and $29 \pm 10$ vs. $24 \pm 7 \mathrm{mmHg}$, respectively). STE-GLS in Group A was significantly worse than that in Group B $(-7.6 \pm 3.0 \%$ vs. $-9.9 \pm 3.2 \%, p=0.01)$. According to receiver operating characteristic curve analysis, STE-GLS of $-7.9 \%$ was the best cut-off value for detection of CMR-LGE (sensitivity, $78 \%$; specificity, $74 \%$; area under the curve, 0.74 ).

\section{Conclusions}

STE-GLS may be used as a surrogate marker for the detection of CMR-LGE in patients with NICM.

\section{Introduction}

Nonischemic cardiomyopathy (NICM) is a common phenotype of cardiomyopathy, with a prevalence of approximately 1 in 2500 patients [1]. Adverse outcomes are typically observed, and the 10-year mortality remains over $40 \%$, despite advances in medical devices and pharmacotherapy [2]. Late gadolinium enhancement (LGE) on cardiovascular magnetic resonance (CMR), suggesting myocardial fibrosis is an 
important predictor of mortality, cardiac failure requiring hospitalization, and implantable cardioverter defibrillator (ICD) in patients with NICM [3-10].

Left ventricular (LV) global longitudinal strain (GLS) using speckle-tracking echocardiography (STE) is reportedly a prognostic index in patients with reduced LV ejection fraction (LVEF), including NICM [1114]. The presence of CMR-LGE may be an important factor associated with severely impaired STE-GLS. STE-GLS may be used as a surrogate marker for the detection of CMR-LGE in patients with NICM. However, the significance of STE-GLS for detecting CMR-LGE has not yet been examined in patients with NICM. Therefore, we investigated whether STE-GLS can be used as surrogate marker for detecting CMRLGE in patients with NICM.

\section{Methods}

\section{Study patients}

Using two-dimensional (2D) echocardiography, we retrospectively enrolled 50 patients with NICM having LVEF of < 50\% in whom both STE and CMR were performed between January 2014 and June 2017 and who did not fit the exclusion criteria. All patients had experienced chronic cardiac failure of at least 12 months, with the typical onset symptoms of this condition, including gradually progressive breathlessness, fatigue, and palpitation. None of the patients in the present study exhibited clinical symptoms or signs of ongoing myocarditis. The presence of severe coronary artery disease (CAD; > 50\% diameter luminal stenosis in any coronary artery) was ruled out in all patients using coronary angiography. Moreover, patients with cardiogenic shock, unstable hemodynamic status, severe renal dysfunction (glomerular filtration rate $<30 \mathrm{ml} / \mathrm{min} / 1.73 \mathrm{~m}^{2}$ ), CMR contraindications (e.g., metal implants), moderate or severe mitral and aortic valve regurgitation or stenosis [15, 16], hypertrophic cardiomyopathy, any infiltrative heart disease, atrial fibrillation, and postcardiac operation were carefully excluded. The present study was approved by the Committee for the Protection of Human Subjects in Research at Wakayama Medical University.

\section{Echocardiographic measurements}

Standard transthoracic echocardiographic examinations were performed in all patients using a Vivid E9 or Vivid 7 digital ultrasound system (GE Medical Systems, Horten, Norway). Echocardiography was performed within 1 week of performing CMR. Images were recorded with $>40$ frames per second. Two cardiac cycles were stored in cineloop format for offline analysis. LV end-diastolic volume index (LVEDVI), LV end-systolic volume index (LVESVI), LVEF, and left atrial volume index (LAVI) were measured using biplane Simpson's rule from the apical four- and two-chamber views, according to the criteria established by the American Society of Echocardiography [17]. The E and A waves were measured based on the mitral inflow profile, assessed in the apical four-chamber view using pulsed-wave Doppler echocardiography, with the sample volume placed at the tips of mitral leaflets during diastole. The e' 
velocity from the septal and lateral mitral valve annuli was measured in the apical four-chamber view using Doppler tissue imaging of the mitral annulus. The systolic transtricuspid pressure gradient (TR-PG) was calculated from the maximal continuous-wave Doppler velocity of the tricuspid regurgitant jet, calculated using the modified Bernoulli equation.

STE-GLS was determined using 2D speckle-tracking strain analysis using an EchoPAC version 113 workstation (GE Medical Systems, Horten, Norway), as described previously [18-20]. This speckletracking software tracks the frame-to-frame movement of speckles (natural acoustic markers) in standard 2D echocardiographic images. The percentage change in length/initial length of the speckle pattern over the cardiac cycle was calculated as the longitudinal strain.

Two-dimensional speckle-tracking strain analysis was performed using standard 2D images of the apical two-chamber, four-chamber, and long-axis view by a certified and experienced cardiologist blinded to the CMR data. After defining the mitral annulus and apex at the end-systolic frame in each view, the software automatically traced the endocardial border, mid-myocardial layer, and epicardial border, including the entire myocardium. The width of the region of interest (ROI) was manually adjusted to ensure accurate tracking of the myocardial wall. The software automatically tracked speckles throughout the cardiac cycle; it accepted segments of good tracking quality and rejected poorly tracked segments. The tracking algorithm facilitates further manual adjustment of ROI for ensuring that all myocardial segments were included throughout the cardiac cycle. After completion of 2D speckle tracking in the three apical views, the results of the LV longitudinal strain analysis were automatically combined in a single bull's eye summary provided the peak systolic longitudinal strain for each LV segment, with the mean peak systolic longitudinal strain value for each view and mean global longitudinal peak systolic strain value for the entire left ventricle (STE-GLS).

A total of 15 patients was randomly selected for assessment of intra- and interobserver reliability of speckle-tracking echocardiographic measurements. The intraobserver analysis was performed by the same observer at two different time points; the observer was blinded to the clinical information and investigation results of the other time point. The interobserver analysis was performed by two observers who were blinded to the clinical information and investigation results. We evaluated the intra- and interobserver reliability of speckle-tracking echocardiographic measurements using intraclass correlation coefficient (ICC).

\section{CMR}

All CMR examinations were performed using a 1.5-T clinical scanner (Intera Achieva; Philips Medical Systems, Best, the Netherlands), equipped with a 32-element cardiac phased-array coil for signal reception, as previously described. During the examination, patients were continuously monitored using single-lead electrocardiography, repeated blood pressure measurements, and pulse oximetry. With the patient in the supine position, contiguous short-axis cine images encompassing the left ventricle from 
base to apex were acquired using a standard steady-state free precession sequence. LGE imaging covering the entire ventricle was performed 10-15 min after the intravenous injection of $0.1 \mathrm{mmol} / \mathrm{kg}$ gadolinium diethylenetriamine penta-acetic acid (Gd-DTPA; Magnevist, Schering, Berlin, Germany). We used a three-dimensional inversion recovery turbo gradient echo sequence, and images were obtained during an end-expiratory breath hold. Scan parameters were as follows: TR, $4.1 \mathrm{~ms}$; TE, $1.25 \mathrm{~ms}$; flip angle, $15^{\circ}$; FOV, $350 \times 350 \mathrm{~mm}$; partial echo; matrix, $224 \times 256$; and spatial resolution, $1.56 \times 2.24 \times 10$ $\mathrm{mm}^{3}$ reconstructed to $0.68 \times 0.68 \times 5 \mathrm{~mm}^{3}$. The inversion time was adjusted to nullify the signal from viable myocardium [21].

All analyses were performed by consensus of the certified and experienced cardiologists blinded to the echocardiographic data on an offline workstation (View Forum, Philips Medical System). According to the previous studies [22,23], the area of LGE was defined as the area with a signal intensity of 5 SDs above the mean signal obtained in the normal myocardium on LGE images.

\section{Statistical analysis}

All statistical analyses were conducted using JMP Pro 13 (SAS Institute Inc., Cary, NC, USA). Categorical variables, presented as frequency counts and percentages, were compared using the Fsher's exact test. Distribution of the continuous data was assessed using the Shapiro-Wilk test. Normally distributed variables were analyzed using the Student's $t$-test, whereas abnormally distributed variables were analyzed using the Wilcoxon test. Receiver operating characteristic (ROC) curve analysis was performed to establish STE-GLS as a predictor of the presence of CMR-LGE. The resulting sensitivity, specificity, and the area under the curve (AUC) were calculated. The best threshold value was determined by the maximum sum of sensitivity and specificity. A p value of $<0.05$ was considered statistically significant.

\section{Results}

\section{Patient clinical characteristics}

The presence, or absence of CMR-LGE was successfully analyzed in all patients with NICM. However, the assessment of STE-GLS in 9 (18\%) of 50 patients was difficult because of poor tracking of the myocardium in > $3 \mathrm{LV}$ segments. Following their exclusion, the final study population comprised 41 patients, for whom both CMR-LGE and STE-GLS analyses were successful. We divided the final study populations into two groups: 18 patients with CMR-LGE (Group A) and 23 without CMR-LGE (Group B). Patients' clinical characteristics of the final cohort are summarized in Table 1. Hypertension and diabetes mellitus were present in $23(56 \%)$ and $5(12 \%)$ patients, respectively. Most patients were treated with angiotensin-converting enzyme inhibitors or angiotensin receptor blockers (98\%), beta blockers (78\%), and furosemide (68\%). No significant differences in patient clinical characteristics were observed between both groups. 


\section{Comparison of echocardiographic findings between both groups}

The echocardiographic parameters of the final study population are summarized in Table 2. No significant differences were observed in LVEDVI, LVESVI, mitral E/A, deceleration time, E/e', LAVI, and systolic transtricuspid pressure gradient between the Groups A and B (113 \pm 30 vs. $109 \pm 24 \mathrm{~mL} / \mathrm{m}^{2}, 82 \pm$ 27 vs. $78 \pm 22 \mathrm{~mL} / \mathrm{m}^{2}, 1.3 \pm 1.1$ vs. $1.2 \pm 0.8,201 \pm 82$ vs. $214 \pm 69 \mathrm{~ms}, 11.3 \pm 3.7$ vs. $11.7 \pm 5.9,49 \pm 24$ vs. $49 \pm 13 \mathrm{~mL} / \mathrm{m}^{2}$, and $29 \pm 10$ vs. $24 \pm 7 \mathrm{mmHg}$, respectively).

\section{Comparison of STE-GLS between patients with and without CMR-LGE}

The intra- and interobserver reliabilities of STE-GLS were $\operatorname{ICC}(1,2)=0.98$ and $\operatorname{ICC}(2,2)=0.92$, respectively. STE-GLS in Group A was significantly impaired compared with that in Group B $(-7.6 \pm 3.0 \%$ vs. $-9.9 \pm 3.2 \%, p=0.01$, Table 2, Figure 1-A). According to ROC curve analysis, STE-GLS of $-7.9 \%$ was determined as the best threshold value for identifying the presence of CMR-LGE (sensitivity, 78\%; specificity, 74\%; AUC, 0.74) (Figure 2-A). On the other hand, there was no significant difference in LVEF between both groups ( $28 \pm 6$ vs. $29 \pm 7 \%, p=0.70$ ) (Table 2, Figure 1-B), and the ROC curve analysis showed that LVEF of $35 \%$ was the best threshold value for detection of CMR-LGE (sensitivity $89 \%$, specificity $31 \%$, AUC 0.53) (Figure 2-B).

\section{Discussion}

In the present study, we investigated the significance of STE-GLS for the detection of CMR-LGE in patients with NICM. The major study findings were as follows: 1) STE-GLS was worse in patients with NICM who have CMR-LGE than those without CMR-LGE, whereas no significant differences were observed in conventional echocardiographic measurements, such as LVESVI, LVEDVI and LVEF, between both groups. 2) STE-GLS of $-7.9 \%$ was found to be the best threshold value for identifying CMR-LGE, and the ROCrelated AUC of 0.74 confirmed a moderate performance of the test for the detection of LGE. To the best of our knowledge, this is the first study investigating the threshold value of STE-GLS for the detection of CMR-LGE in patients with NICM.

\section{LGE-determined myocardial fibrosis and prognosis in patients with NICM}

Areas of CMR-LGE correspond to those of myocardial fibrosis on histology, and approximately $30 \%$ of patients with NICM have a characteristic linear CMR-LGE in the mid-wall of the septum $[3,24]$. Patients with NICM who have CMR-LGE in the present study exhibited typical mid-wall LGE. Myocardial fibrosis provides a substrate for ventricular re-entrant arrhythmia [6] and is independently associated with an increased risk of mortality and cardiac failure morbidity in patients with NICM [4, 5, 7-10]. Moreover, its 
presence and extent in LV myocardium assessed by CMR-LGE in patients with NICM, substantially determines the likelihood of LV reverse remodeling in response to pharmacological therapy $[25,26]$ and cardiac resynchronization therapy [27].

\section{Detection of CMR-LGE in patients with NICM using STE-GLS}

STE is emerging as a novel index for the assessment of LV mechanics via the quantification of active myocardial longitudinal deformation (STE-GLS). Severely impaired STE-GLS is reportedly a prognostic index in patients with reduced LVEF, including those with NICM [11-14]. However, the reason for severely impaired STE-GLS being a prognostic predictor has not yet been clearly explained. We hypothesized that myocardial fibrosis contributes to severely impaired STE-GLS. The best threshold value of STE-GLS of 7.9\% for identifying CMR-LGE, which suggests myocardial fibrosis, corresponds to the STE-GLS threshold values for identifying worse prognosis reported in previous studies $[11,12]$. This finding suggests that severely impaired STE-GLS as a prognostic predictor is associated with the presence of myocardial fibrosis.

LVEF was generally reported as a strong prognostic predictor, however there was no significant difference in LVEF between the patients with CMR-LGE and those without CMR-LGE. Moreover, according to the ROC curve analysis for identifying CMR-LGE by LVEF, the AUC of 0.53 showed a low performance of the test for the detection of CMR-LGE. These results suggest that it is difficult to identify CMR-LGE by measurement of LVEF, and that LVEF cannot be used as a surrogate marker for the detection of CMR-LGE in patients with NICM.

In this study, there were six patients with severely impaired STE-GLS (STE-GLS > -7.9\%) in whom CMRLGE was not found. CMR-LGE cannot depict diffuse myocardial fibrosis because CMR-LGE is based on the null point method. In patients without CMR-LGE, there might be some patients with diffuse myocardial fibrosis in whom STE-GLS might be severely impaired.

\section{Advantages of STE-GLS in NICM compared with CMR}

First, STE-GLS can be used in patients with NICM having renal dysfunction. The use of gadolinium contrast is limited in patients with renal dysfunction in CMR-LGE examinations. Therefore, another modality that does not require contrast agents for identifying myocardial fibrosis is required. Noninvasive STE-GLS without contrast agents may be used as an ideal modality for detecting myocardial fibrosis. Second, it is possible to estimate STE-GLS in patients in whom CMR-LGE examinations could not be completed because of the patient's intolerance to staying in the CMR examination room or difficulty in holding their breath during CMR-LGE examinations. Finally, the present results contribute to the daily clinical practices associated with the management of NICM, even in the institutes where CMR is not available. STE-GLS, which is an established index and is measured rather straightforwardly in echocardiographic laboratories, would be helpful for decision-making in the management of NICM.

\section{Study limitations}


This study has several limitations. First, this was a retrospective observational study, with a relatively small sample size. A prospective multicenter study with large sample size should be conducted in future. Second, STE-GLS measurements are dependent on echocardiographic image quality. Accordingly, STEGLS measurement was unsuccessful in $18 \%$ of the initial study cohort. ROI of the speckle-tracking software may be challenging to fit strain analyses in patients with NICM patients owing to the thin wall, which could have influenced our data. In addition, the definition of successful measurements of STE-GLS may be too strict in this study. In some previous studies evaluating STE-GLS, the overall STE-GLS was calculated in only two of three apical images when only three apical images could be assessed because of poor tracking of ROI $[13,28,29]$. This alternative method should be considered for the clinical application of STE-GLS in patients with NICM.

\section{Conclusions}

STE-GLS may be used as a surrogate marker for the detection of CMR-LGE in patients with NICM.

\section{Abbreviations}

ACEI

Angiotensin converting enzyme inhibitor

ARB

Angiotensin receptor blocker

AUC

area under the curve

BNP

Brain natriuretic peptide

CAD

coronary artery disease

CMR

cardiovascular magnetic resonance

DT

deceleration time

EDVI

end-diastolic volume index

EF

ejection fraction

ESVI

end-systolic volume index

eGFR

Estimated glomerular filtration rate

GLS 
global longitudinal strain

ICC

intraclass correlation coefficient

ICD

implantable cardioverter defibrillator

LAVI

and left atrial volume index

LGE

late gadolinium enhancement

LV

left ventricular

LVDD

Left ventricular dimension diastole

LVDS

Left ventricular dimension systole

$\mathrm{NICM}$

nonischemic cardiomyopathy

NYHA

New York Heart Association Class

STE

speckle-tracking echocardiography

TR-PG

systolic transtricuspid pressure gradient

ROC

receiver operating characteristic

$\mathrm{ROI}$

region of interest

\section{Declarations}

Acknowledgments: Not applicable.

Authors' contributions: SO, TT and TH designed and drafted the manuscript. KT, TH, TT, SO, TW, MK, AK, $\mathrm{YM}, \mathrm{HK}$ and $\mathrm{YI}$ acquired data and performed measurements. KS and YS supported the statistical analysis. TH, TK, AT and TA substantially contributed to the manuscript and revised it critically for important intellectual content. All authors approved the submitted version.

Funding: This work has not received any kind of financial support.

Availability of data and materials: The dataset analyzed during the current study are available from the corresponding author on reasonable request. 
Ethics approval and consent to participate: Informed written consent was obtained from all participants in this study. The study protocol was approved by the Ethics Committee of Wakayama Medical University (No. 2141) and the study has been performed in accordance with the Declaration of Helsinki.

Consent for publication: Not applicable.

Competing interests: The authors declare that they have no competing interests.

Author's information: See title page

\section{References}

1. Jefferies JL, Towbin JA. Dilated cardiomyopathy. Lancet. 2010;375:752-62.

2. Felker GM, Thompson RE, Hare JM, Hruban RH, Clemetson DE, Howard DL, et al. Underlying causes and long-term survival in patients with initially unexplained cardiomyopathy. $\mathrm{N}$ Engl $\mathrm{J}$ Med. 2000;342:1077-84.

3. McCrohon JA, Moon JC, Prasad SK, McKenna WJ, Lorenz CH, Coats AJ, et al. Differentiation of heart failure related to dilated cardiomyopathy and coronary artery disease using gadolinium-enhanced cardiovascular magnetic resonance. Circulation. 2003;108:54-9.

4. Assomull RG, Prasad SK, Lyne J, Smith G, Burman ED, Khan M, et al. Cardiovascular magnetic resonance, fibrosis, and prognosis in dilated cardiomyopathy. J Am Coll Cardiol. 2006;48:1977-85.

5. Wu KC, Weiss RG, Thiemann DR, Kitagawa K, Schmidt A, Dalal D, et al. Late Gadolinium Enhancement by Cardiovascular Magnetic Resonance Heralds an Adverse Prognosis in Nonischemic Cardiomyopathy. J Am Coll Cardiol. 2008;51:2414-21.

6. Iles L, Pfluger $H$, Lefkovits L, Butler MJ, Kistler PM, Kaye DM, et al. Myocardial fibrosis predicts appropriate device therapy in patients with implantable cardioverter defibrillators for primary prevention of sudden cardiac death. J Am Coll Cardiol. 2011;57:821-8.

7. Lehrke S, Lossnitzer D, Schöb M, Steen H, Merten C, Kemmling H, et al. Use of cardiovascular magnetic resonance for risk stratification in chronic heart failure: prognostic value of late gadolinium enhancement in patients with non-ischemic dilated cardiomyopathy. Heart. 2011;97:727-32.

8. Neilan TG, Coelho-Filho OR, Danik SB, Shah RV, Dodson JA, Verdini DJ, et al. CMR Quantification of Myocardial Scar Provides Additive Prognostic Information in Nonischemic Cardiomyopathy. J Am Coll Cardiol Img. 2013;6:944-54.

9. Gulati A, Jabbour A, Ismail TF, Guha K, Khwaja J, Raza S, et al. Association of fibrosis with mortality and sudden cardiac death in patients with nonischemic dilated cardiomyopathy. JAMA. 2013;309:896-908.

10. Kuruvilla S, Adenaw N, Katwal AB, Lipinski MJ, Kramer CM, Salerno M. Late gadolinium enhancement on cardiac magnetic response predicts adverse cardiovascular outcomes in noninvasive cardiomyopathy: A systematic review and meta-analysis. Circ Cardiovasc Imaging. 2014;7:250-8. 
11. Mignot A, Donal E, Zaroui A, Reant P, Salem A, Hamon C, et al. Global longitudinal strain as a major predictor of cardiac events in patients with depressed left ventricular function: a multicenter study. $\mathrm{J}$ Am Soc Echocardiogr. 2010;23:1019-24.

12. Motoki H, Borowski AG, Shrestha K, Troughton RW, Tang WH, Thomas JD, et al. Incremental prognostic value of assessing left ventricular myocardial mechanics in patients with chronic systolic heart failure. J Am Coll Cardiol. 2012;60:2074-81.

13. Sengeløv M, Jørgensen PG, Jensen JS, Bruun NE, Olsen FJ, Fritz-Hansen T, et al. Global longitudinal strain is a superior predictor of all-cause mortality in heart failure with reduced ejection fraction. $J$ Am Coll Cardiol Img. 2015;8:1351-9.

14. Chimura M, Onishi T, Tsukishiro Y, Sawada T, Kiuchi K, Shimane A, et al. Longitudinal strain combined with delayed-enhancement magnetic resonance improves risk stratification in patients with dilated cardiomyopathy. Heart. 2017;103:679-86.

15. Zoghbi WA, Adams D, Bonow RO, Enriquez-Sarano M, Foster E, Grayburn PA, et al. Recommendations for noninvasive evaluation of native valvular regurgitation: a report from the American Society of Echocardiography developed in collaboration with the Society for Cardiovascular Magnetic Resonance. J Am Soc Echocardiogr. 2017;30:303-71.

16. Baumgartner $\mathrm{H}$, Hung J, Bermejo J, Chambers JB, Evangelista A, Griffin BP, et al. Echocardiographic assessment of valve stenosis: EAE/ASE recommendations for clinical practice. J Am Soc Echocardiogr. 2009;22:1-23.

17. Lang RM, Badano LP, Mor-Avi V, Afilalo J, Armstrong A, Ernande L, et al. Recommendations for chamber quantification by echocardiography in adults: an update report from the American Society of Echocardiography and European Association of Cardiovascular Imaging. J Am Soc Echocardiogr. 2015;28:1-39.

18. Delgado V, Mollema SA, Ypenburg C, Tops LF, van der Wall EE, Schalij MJ, et al. Relation between global left ventricular longitudinal strain assessed with novel automated function imaging and biplane left ventricular ejection fraction in patients with coronary artery disease. J Am Soc Echocardiogr. 2008;21:1244-50.

19. Mollema SA, Delgado V, Bertini M, Antoni ML, Boersma E, Holman ER, et al. Viability assessment with global left ventricular longitudinal strain predicts recovery of left ventricular function after acute myocardial infarction. Circ Cardiovasc Imaging. 2010;3:15-23.

20. Bertini M, Ng AC, Antoni ML, Nucifora G, Ewe SH, Auger D, et al. Global longitudinal strain predicts long-term survival in patients with chronic ischemic cardiomyopathy. Circ Cardiovasc Imaging. 2012;5:383-91.

21. Simonetti OP, Kim RJ, Fieno DS, Hillenbrand HB, Wu E, Bundy JM, et al. An improved MR imaging technique for the visualization of myocardial infarction. Radiology. 2001;218:215-23.

22. Judd RM, Lugo-Olivieri CH, Arai M, Kondo T, Croisille P, Lima JA, et al. Physiological basis of myocardial contrast enhancement in fast magnetic resonance images of 2-day-old reperfused canine infarcts. Circulation. 1995;92:1902-10. 
23. Bondarenko O, Beek AM, Hofman MB, Kühl HP, Twisk JW, van Dockum WG, et al. Standardizing the definition of hyperenhancement in the quantitative assessment of infarct size and myocardial viability using delayed contrast-enhanced CMR. J Cardiovasc Magn Reson. 2005;7:481-5.

24. de Leeuw N, Ruiter DJ, Balk AH, de Jonge N, Melchers WJ, Galama JM. Histopathologic findings in explanted heart tissue from patients with end-stage idiopathic dilated cardiomyopathy. Transpl Int. 2001;14:299-306.

25. Leong DP, Chakrabarty A, Shipp N, Molaee P, Madsen PL, Joerg L, et al. Effects of myocardial fibrosis and ventricular dyssynchrony on response to therapy in new presentation idiopathic dilated cardiomyopathy: insights from cardiovascular magnetic resonance and echocardiography. Eur Heart J. 2012;33:640-8.

26. Masci PG, Schuurman R, Andrea B, Ripoli A, Coceani M, Chiappino S, et al. Myocardial fibrosis as a key determinant of left ventricular remodeling in idiopathic dilated cardiomyopathy: a contrastenhanced cardiovascular magnetic study. Circ Cardiovasc Imaging. 2013;6:790-9.

27. Leyva F, Taylor RJ, Foley PW, Umar F, Mulligan LJ, Patel K, et al. Left ventricular midwall fibrosis as a predictor of mortality and morbidity after cardiac resynchronization therapy in patients with nonischemic cardiomyopathy. J Am Coll Cardiol. 2012;60:1659-67.

28. Stanton T, Leano R, Marwick TH. Prediction of all-cause mortality from global longitudinal speckle strain: Comparison with ejection fraction and wall motion score. Circ Cardiovasc Imaging. 2009;2:356-64.

29. Ersbø|l M, Valeur N, Mogensen UM, Andersen MJ, Møller JE, Velazquez EJ, et al. Prediction of allcause mortality and heart failure admissions from global left ventricular longitudinal strain in patients with acute myocardial infarction and preserved left ventricular ejection fraction. J Am Coll Cardiol. 2013;61:2365-73.

\section{Tables}

Table 1. Patient characteristics 


\begin{tabular}{|c|c|c|c|}
\hline & Group A & Group B & p-value \\
\hline & (with CMR-LGE) & (without CMR-LGE) & \\
\hline & $n=18$ & $n=23$ & \\
\hline Age, years & $60 \pm 16$ & $59 \pm 12$ & 0.65 \\
\hline Male & $17(94)$ & $17(74)$ & 0.11 \\
\hline Hypertension & $12(67)$ & $11(50)$ & 0.35 \\
\hline Diabetes mellitus & $1(6)$ & $4(18)$ & 0.36 \\
\hline NYHA & & & 0.05 \\
\hline 1 & $1(5)$ & $7(30)$ & \\
\hline II & $7(40)$ & $6(26)$ & \\
\hline III & $9(50)$ & $5(22)$ & \\
\hline IV & $1(5)$ & $5(22)$ & \\
\hline Creatinine, $\mathrm{mg} / \mathrm{dL}$ & $0.96 \pm 0.26$ & $0.86 \pm 0.26$ & 0.24 \\
\hline $\mathrm{eGFR}, \mathrm{mL} / \mathrm{min} / 1.73 \mathrm{~m}^{2}$ & $68.0 \pm 24.1$ & $72.3 \pm 21.2$ & 0.35 \\
\hline Systolic blood pressure, $\mathrm{mmHg}$ & $136 \pm 27$ & $123 \pm 26$ & 0.12 \\
\hline Diastolic blood pressure, $\mathrm{mmHg}$ & $87 \pm 22$ & $77 \pm 17$ & 0.18 \\
\hline \multicolumn{4}{|l|}{ Medications } \\
\hline Furosemide & $14(78)$ & $14(61)$ & 0.32 \\
\hline ACEI/ARB & $18(100)$ & $22(97)$ & 1.00 \\
\hline Spironolactone & $6(33)$ & $10(43)$ & 0.54 \\
\hline Beta-blocker & $16(89)$ & $16(70)$ & 0.25 \\
\hline Amiodarone & 3 (17) & $3(13)$ & 1.000 \\
\hline BNP, pg/dL & $961.8 \pm 1073.8$ & $652.8 \pm 690.0$ & 0.39 \\
\hline
\end{tabular}


Values are presented as mean \pm SD or $n(\%) . C M R-L G E=$ Late gadolinium enhancement on cardiovascular magnetic resonance; NYHA = New York Heart Association Class; eGFR = Estimated glomerular filtration rate; $\mathrm{ACEI}=$ Angiotensin converting enzyme inhibitor; $\mathrm{ARB}=$ Angiotensin receptor blocker; $\mathrm{BNP}=$ Brain natriuretic peptide

Table 2. Echocardiography findings

\begin{tabular}{|c|c|c|c|}
\hline & $\begin{array}{l}\text { Group A } \\
\text { (with CMR-LGE) }\end{array}$ & $\begin{array}{l}\text { Group B } \\
\text { (without CMR-LGE) }\end{array}$ & $\mathrm{p}$-value \\
\hline & $n=18$ & $n=23$ & \\
\hline LVDD, mm & $60 \pm 8$ & $61 \pm 7$ & 0.58 \\
\hline LVDS, mm & $53 \pm 9$ & $53 \pm 7$ & 0.86 \\
\hline LVEDVI, $\mathrm{mL} / \mathrm{m}^{2}$ & $113 \pm 30$ & $109 \pm 24$ & 0.97 \\
\hline LVESVI, $\mathrm{mL} / \mathrm{m}^{2}$ & $82 \pm 27$ & $78 \pm 22$ & 0.89 \\
\hline LVEF, \% & $28 \pm 6$ & $29 \pm 7$ & 0.70 \\
\hline $\mathrm{LAVI}, \mathrm{mL} / \mathrm{m}^{2}$ & $49 \pm 24$ & $49 \pm 13$ & 0.91 \\
\hline E/A ratio & $1.3 \pm 1.1$ & $1.2 \pm 0.8$ & 0.60 \\
\hline $\mathrm{DT}, \mathrm{ms}$ & $201 \pm 82$ & $214 \pm 69$ & 0.43 \\
\hline E/e' mean ratio & $11.3 \pm 3.7$ & $11.7 \pm 5.9$ & 0.85 \\
\hline TR-PG, mmHg & $29 \pm 10$ & $24 \pm 7$ & 0.16 \\
\hline STE-GLS, \% & $-7.6 \pm 3.0$ & $-9.9 \pm 3.2$ & 0.01 \\
\hline
\end{tabular}


Values are presented as mean \pm SD. CMR-LGE = Late gadolinium enhancement on cardiovascular magnetic resonance; LVDD = Left ventricular dimension diastole; LVDS = Left ventricular dimension systole; LVEDVI = Left ventricular end diastolic volume index; LVESVI = Left ventricular end systolic volume index; $L V E F=$ Left ventricular ejection fraction; $L A V I=$ Left atrial volume index; $D T=$ Deceleration time; TR-PG = systolic transtricuspid pressure gradient; STE-GLS = global longitudinal strain using speckle-tracking echocardiography.

\section{Figures}
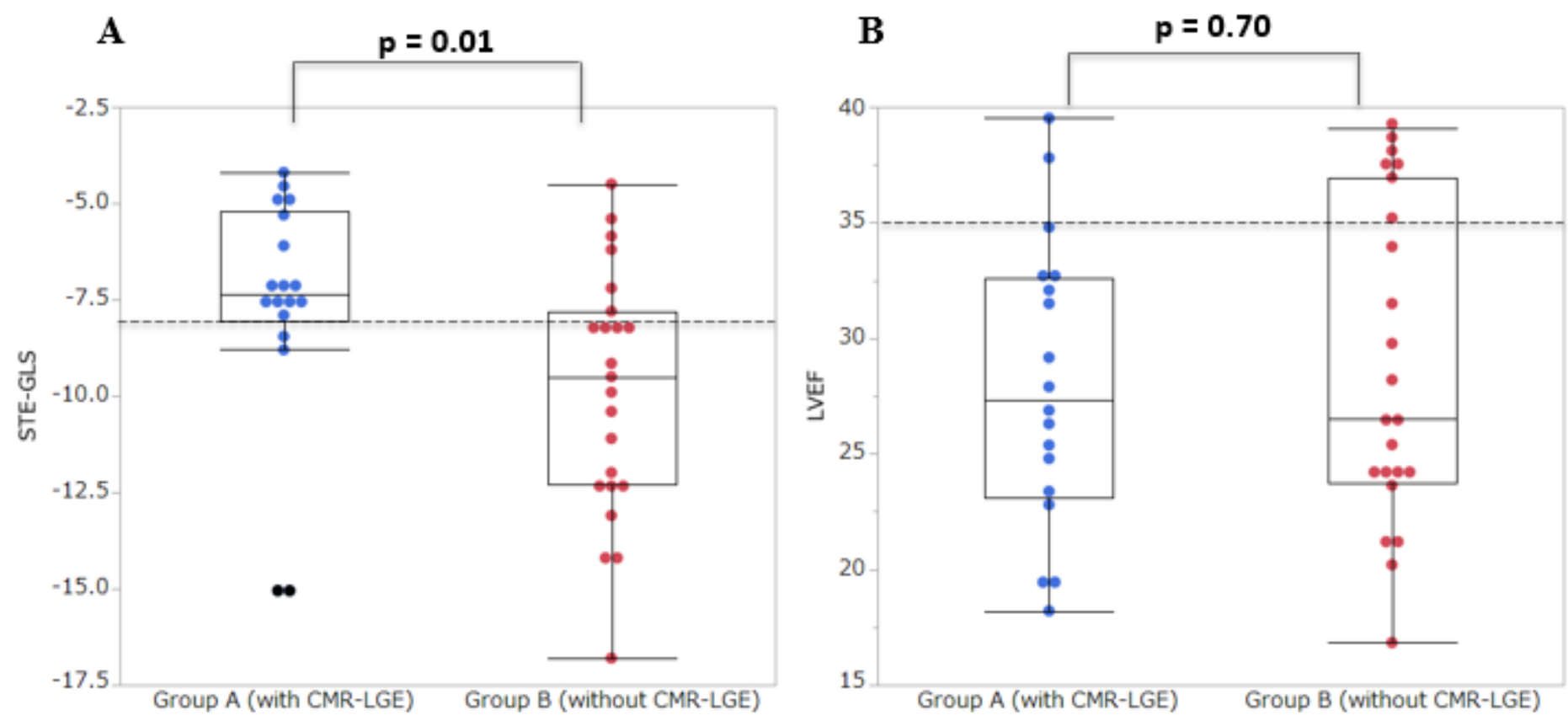

Figure 1

Relationship between STE-GLS and CMR-LGE (A), and that between LVEF and CMR-LGE (B). STE-GLS was significantly worse in patients with CMR-LGE than in those without CMR-LGE. On the other hand, there was no significant difference in LVEF. The broken lines present the cutoff values of LVGLS (-7.9\%) and LVEF (35\%) for detecting CMR-LGE. STE-GLS = Global longitudinal strain assessed by speckletracking echocardiography; CMR-LGE = Late gadolinium enhancement on cardiovascular magnetic resonance; LVEF = left ventricular ejection fraction. 
$\mathbf{A}$

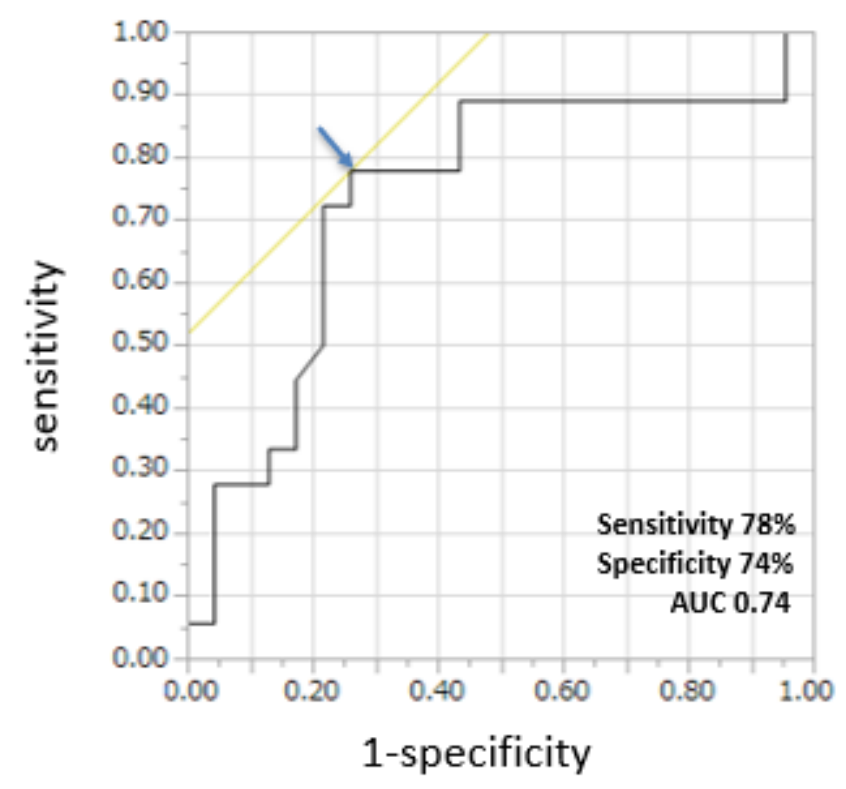

B

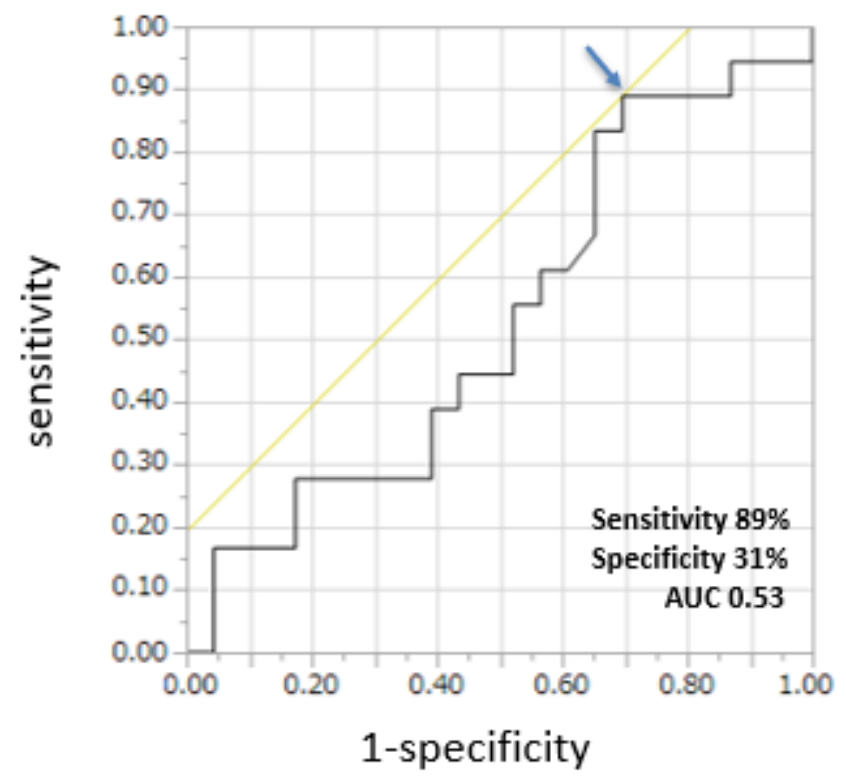

Figure 2

ROC curves of STE-GLS (A) and LVEF (B) for the detection of the presence of CMR-LGE. STE-GLS of $-7.9 \%$ and LVEF of $35 \%$ are determined as the best threshold values for identifying CMR-LGE (sensitivity $78 \%$, specificity $74 \%$, AUC 0.74 , and sensitivity $89 \%$, specificity $31 \%$, AUC 0.53 , respectively). ROC = Receiver Operating Characteristic; STE-GLS = Global longitudinal strain assessed by speckle-tracking echocardiography; $\mathrm{LVEF}=$ Left ventricular ejection fraction; $C M R-L G E=$ Late gadolinium enhancement on cardiovascular magnetic resonance; $\mathrm{AUC}=$ Area under the curve. 